\title{
INDICADORES PARA GESTÃO DE RESÍDUOS SÓLIDOS URBANOS: CARACTERIZAÇÃO DO GRUPO DE ESPECIALISTAS
}

Tatiane Bonametti Veiga ${ }^{1}$

Sílvia Carla da Silva André ${ }^{2}$

\section{Angela Maria Magosso Takayanagui ${ }^{3}$}

RESUMO: Os indicadores, em essência, são importantes ferramentas de apoio à decisão, devendo ser elaborado segundo as necessidades dos potenciais usuários. No campo de gestão de resíduos, os indicadores podem auxiliar os gestores nas ações que devem ser realizadas a fim de proporcionar melhores condições para a sociedade, e consequentemente uma maior qualidade de vida e saúde para as pessoas. Na elaboração desses indicadores é necessária à participação de especialistas na área da investigação, sendo assim, o objetivo deste estudo consiste em levantar e caracterizar um grupo de especialistas na área de resíduos para a construção de indicadores na gestão de resíduos sólidos urbanos. Os achados revelaram que pesquisadores das diferentes regiões do Brasil desenvolvem pesquisas na área de resíduos sólidos em grupos de pesquisas em vários estados brasileiros. Destaca-se que foi encontrada uma maior representatividade na região nordeste e sudeste de grupos de pesquisa que direcionam seus estudos nessa área. Entre os especialistas foi observada uma formação predominante na área das engenharias, mas com uma multidisciplinaridade extensa e inusitada no grupo, fator esse que pode proporcionar um

\footnotetext{
${ }^{1}$ Doutoranda em Ciências, Programa de Pós-graduação em Enfermagem em Saúde Pública, Universidade de São Paulo - USP. Engenheira Civil. tati.veiga@yahoo.com.br.

${ }^{2}$ Doutoranda em Ciências, Programa de Pós-graduação em Enfermagem em Saúde Pública, Universidade de São Paulo - USP. Enfermeira. silviacarlabjp@gmail.com.br.

${ }^{3}$ Doutora em Enfermagem. Universidade de São Paulo - USP. Professora Associada da Escola de Enfermagem de Ribeirão Preto (EERP/USP). amtakay@eerp.usp.br.
} 
enriquecimento na construção de novos indicadores. Dessa forma, conclui-se que a questão abordada nesta investigação não é uma preocupação restrita a áreas específicas ou apenas a determinadas localidades ou instituições, mas extrapola essas delimitações por ser um problema extensivo a toda sociedade.

Palavras-chave: Indicadores. Resíduos Sólidos. Pesquisadores.

\section{INTRODUÇÃO}

O termo "indicador" origina-se do latim indicare que significa apontar para, desvendar, estimar ou trazer ao conhecimento público (OLIVEIRA; FARIA, 2008). Em 1992, a Agenda 21 já indicava, em seu capítulo 40, a importância na elaboração de indicadores sob o paradigma do desenvolvimento sustentável (CNUMAD, 2001).

Segundo Meadows (1998), os indicadores devem ser claros, viáveis, suficientes, oportunos, democráticos e ter relevância política. Bossel (1999) ainda afirma que indicadores devem representar um conjunto de preocupações relevantes, capazes de inferir a viabilidade e sustentabilidade de desenvolvimentos correntes, preocupando-se com os impactos que podem gerar, direta ou indiretamente, em diferentes áreas do conhecimento e da sociedade.

Os indicadores devem ser facilmente aplicáveis pelo usuário, ter uma relação custo-benefício favorável e sua aplicabilidade deve ser geral e não voltada a uma questão específica, destacando a importância de ser cientificamente sólida, imparcial, confiável em termos de ciência e não vulnerável a pequenas mudanças (PAPINI, 2012).

Um papel central pode ser desempenhado pelos indicadores por possibilitar sua utilização "como ferramenta de mobilização das partes interessadas na análise e avaliação da sustentabilidade do desenvolvimento, bem como nos processos de educação e comunicação" (MALHEIROS; COUTINHO; PHILIPPI JR, 2013).

Orientações para o desenvolvimento de indicadores de sustentabilidade são apresentadas resumidamente na terceira edição de Indicadores de Desenvolvimento Sustentável: Diretrizes e Metodologias, editado pela Organização das Nações Unidas. As diretrizes recomendadas, neste documento, indica a prioridade no âmbito nacional; a relevância para avaliar o progresso do desenvolvimento sustentável; a elaboração em 
número limitado, mas com possibilidades em aberto e adaptável às necessidades futuras; a ampla cobertura da Agenda 21 e de todos os aspectos do desenvolvimento sustentável; ser compreensível, claro e inequívoco; conceitualmente aceitável; na medida do possível ser representativo no consenso internacional; dentro das capacidades de desenvolvimento dos governos nacionais e dependente de dados relativos a custoefetividade (UNITED NATIONS, 2007).

No curso da história, a construção de indicadores foi inicialmente voltada ao setor econômico, como o cálculo do Produto Interno Bruto, na década de 40 (MEADOWS, 1998). Somente na década de 70, começou a haver amplas discussões em relação à elaboração de indicadores voltados para a sustentabilidade, surgindo modelos fundamentados em outras dimensões, além da econômica, como a ambiental, social e institucional (GUIMARÃES; FEICHAS, 2009; MALHEIROS; PHILIPPI JR; COUTINHO, 2008). Muitos indicadores elaborados segundo essas dimensões apresentam implicações, diretas ou indiretas referentes à área da saúde.

Em 1996, na Itália, foram elaborados os Princípios de Bellagio que correspondem a dez diretrizes para selecionar e construir indicadores: guia de visões e metas; elementos essenciais; escopo adequado; foco prático; abertura e transparência; comunicação efetiva; ampla participação; avaliação constante e capacidade institucional, sendo indicada como ponto inicial para análise da sustentabilidade, a pesquisa bibliográfica, a fim de auxiliar na percepção da relação entre sociedade e natureza (INTERNATIONAL INSTITUTE SUSTAINABLE DEVELOPMENT, 2013; RABELO; LIMA, 2007).

Para Foschini (2011) os indicadores devem ser adotados para medir as mudanças em diferentes dimensões no desenvolvimento de sistemas de monitoramento e avaliação na busca pela sustentabilidade.

A construção de indicadores pode auxiliar na gestão pública em diferentes setores da sociedade, destacando, neste estudo, a importância de indicadores na área da gestão de resíduos sólidos como ferramentas que possibilitam o levantamento de dados essenciais para a elaboração, implantação e acompanhamento do Plano Municipal de Gestão Integrada de Resíduos Sólidos (PMGIRS).

Além disso, os indicadores de sustentabilidade proporcionam uma visão abrangente da situação da geração e gerenciamento dos resíduos, podendo ser utilizado para direcionar a escolha das melhores tecnologias e técnicas mais adequadas para cada 
tipo de resíduo gerado nos municípios, contribuindo para que os recursos financeiros possam ser direcionados em um processo sustentável.

Tendo em vista as exigências apresentadas na Política Nacional de Resíduos Sólidos (PNRS) (BRASIL, 2010), surge a necessidade da realização de novas pesquisas no setor de resíduos sólidos urbanos, voltadas à elaboração de indicadores que possam trazer subsídios para aplicação dessa lei, contribuindo para a fundamentação de estratégias sólidas na busca pelo desenvolvimento sustentável.

Assim, este estudo tem como objetivo apresentar a caracterização de um grupo de especialistas na área de gestão de resíduos sólidos que participam de uma pesquisa para a construção de uma lista de indicadores para a gestão de resíduos, com base nas diretrizes da PNRS, à luz de suas implicações na área da saúde, sob a égide dos princípios de desenvolvimento sustentável.

\section{METODOLOGIA}

Para identificação de especialistas na área de gestão de resíduos sólidos foi realizado um levantamento junto à base do Conselho Nacional do Desenvolvimento Científico e Tecnológico (CNPq), buscando coordenadores de grupos com linhas de pesquisa relacionadas à área de gestão de resíduos sólidos.

A partir dessa lista, foi realizado um levantamento fundamentado no currículo lattes dos especialistas, a fim de verificar a produção na área desta investigação e sua atuação em pesquisas realizadas nas instituições de ensino.

Decorrido esse processo, foram selecionados os especialistas aos quais seria encaminhado o convite para participação da pesquisa. Com o objetivo de efetuar esse contato foi realizado um levantamento prévio para obter o e-mail e telefone das instituições de ensino as quais estavam vinculados.

Após essa trajetória, foi encaminhado um e-mail a todos os líderes dos grupos de pesquisa identificados junto à base do CNPq, informando sobre a importância de sua participação, tanto para esta pesquisa, quanto para a área de conhecimento. No e-mail 
constava a Carta Convite com a explicação da pesquisa, o Termo de Consentimento Livre e Esclarecido (TCLE) e a Ficha de Identificação dos sujeitos.

Frente à impossibilidade de participação de alguns pesquisadores convidados no estudo, foi utilizada a estratégia denominada "snowball" (FREITAS et al., 2000), solicitando aos coordenadores dos grupos de pesquisa que indicassem outros especialistas de seu grupo com produções na área de gestão de resíduos sólidos que pudessem trazer contribuições significativas na construção de indicadores de sustentabilidade para a gestão de resíduos sólidos, segundo as diretrizes da PNRS.

Para realizar o convite aos novos especialistas indicados pelos coordenadores, foi utilizado o mesmo procedimento descrito: análise do currículo lattes, busca de e-mails e contatos telefônicos, identificação da instituição de ensino que estavam vinculados e encaminhamento do e-mail com os referidos documentos anexos.

Aos especialistas que não se pronunciaram após o prazo determinado, foi realizado contato telefônico para reforçar a importância das suas contribuições para 0 desenvolvimento da pesquisa. Esse contato mais direto visou maximizar o índice de participação dos especialistas.

Esta pesquisa só teve início após aprovação Comitê de Ética em Pesquisa da Escola de Enfermagem de Ribeirão Preto da Universidade de São Paulo.

\section{CARACTERIZAÇÃO DO GRUPO DE ESPECIALISTAS}

Com relação à seleção dos sujeitos desta pesquisa, foi utilizado como critério sua experiência na área de gestão de resíduos, mas não foi delimitado uma homogeneidade em suas formações acadêmicas. Buscou-se, dessa forma, uma multidisciplinaridade que possibilita a verificação de diferentes visões de especialistas no processo de construção dos indicadores.

A partir do levantamento realizado junto à base corrente do Diretório dos Grupos de Pesquisa do CNPq foi possível obter uma representatividade de grupos, nas diferentes regiões do Brasil, que direcionam suas pesquisas para discutir fatores que influenciam na gestão de resíduos sólidos. A participação de pesquisadores de diversos estados 
brasileiros consiste em um dos focos desse estudo por trazer para a discussão diferentes realidades, o que possibilita um enriquecimento na construção de novos indicadores.

Foram identificados 120 grupos que atendiam a busca de resíduos sólidos urbanos/resíduos sólidos e possuíam linhas de pesquisas relacionadas à gestão de resíduos.

Com a definição dos grupos foram analisados os currículos de seus coordenadores. De acordo com esse levantamento foi verificado uma maior representatividade de pesquisadores que atuam na área de gestão de resíduos vinculados a grupos de pesquisa na região Nordeste (34,2\%), e a menor representatividade na região Norte (5,0\%). Após o convite realizado a todos os pesquisadores identificados, obteve-se um maior número de participação de sujeitos na região Nordeste $(45,1 \%)$ (Tabela 1$)$.

Tabela 1 - Distribuição dos sujeitos convidados/participantes, segundo divisões regionais no Brasil.

\begin{tabular}{lcccc}
\hline $\begin{array}{c}\text { Região do } \\
\text { Brasil }\end{array}$ & $\begin{array}{c}\text { No de sujeitos } \\
\text { convidados }\end{array}$ & (\%) & $\begin{array}{c}\text { № de participantes } \\
\text { efetivos }\end{array}$ & (\%) \\
\hline Norte & 6 & 5,0 & 3 & 2,5 \\
Nordeste & 41 & 34,2 & 23 & 19,2 \\
Centro-Oeste & 9 & 7,5 & 1 & 0,8 \\
Sudeste & 36 & 30,0 & 15 & 12,5 \\
Sul & 28 & 23,3 & 10 & 8,3 \\
Total & $\mathbf{1 2 0}$ & $\mathbf{1 0 0}$ & $\mathbf{5 2}$ & $\mathbf{4 3 , 3}$ \\
\hline
\end{tabular}

Destaca-se que, dos 120 pesquisadores na área de resíduos convidados para a participação na pesquisa, ocorreu à participação efetiva de 52 sujeitos, que representa $43,3 \%$ dos especialistas convidados. Os sujeitos que participaram da pesquisa preencheram uma Ficha de Identificação que possibilitou elaborar um perfil desses especialistas na área de gestão de resíduos.

Entre esses sujeitos, detectou-se que $27(51,9 \%)$ eram do sexo masculino e 25 $(48,1 \%)$ do sexo feminino, com uma média de idade de 47 anos, que variou de 29 a 70 anos. Os especialistas encontravam-se vinculados tanto a instituições Federais $(53,8 \%)$, Estaduais (21,2\%), Tecnológicas $(3,8 \%)$ e Particulares $(21,2 \%)$. 
Como foi utilizada a técnica de "snowball" solicitando aos coordenadores que indicassem outros pesquisadores de seu grupo com experiência na área, detectou-se uma participação de $76,9 \%$ de coordenadores e $23,1 \%$ de pesquisadores dos grupos de pesquisa.

Os achados revelaram que os grupos de pesquisa os quais esses especialistas estavam vinculados encontravam-se distribuídos em 17 diferentes estados de federação no Brasil, com uma maior concentração no estado de São Paulo (15,8\%), conforme representado na Tabela 2 .

Tabela 2 - Distribuição dos especialistas, segundo os estados da federação - Brasil $(n=52)$

\begin{tabular}{lcc}
\hline Estado & № de participantes & $\mathbf{( \% )}$ \\
Acre & 1 & $1,9 \%$ \\
Tocantins & 2 & $3,8 \%$ \\
Alagoas & 1 & $1,9 \%$ \\
Bahia & 6 & $11,5 \%$ \\
Ceara & 3 & $5,8 \%$ \\
Paraíba & 5 & $9,6 \%$ \\
Pernambuco & 5 & $9,6 \%$ \\
Rio Grande do Norte & 2 & $3,8 \%$ \\
Sergipe & 1 & $1,9 \%$ \\
Distrito Federal & 1 & $1,9 \%$ \\
Espírito Santo & 1 & $1,9 \%$ \\
Minas Gerais & 2 & $3,8 \%$ \\
Rio de Janeiro & 3 & $5,8 \%$ \\
São Paulo & 8 & $15,4 \%$ \\
Paraná & 7 & $13,5 \%$ \\
Santa Catarina & 2 & $3,8 \%$ \\
Rio Grande do Sul & 2 & $3,8 \%$ \\
Total & 52 & $\mathbf{1 0 0 , 0 \%}$ \\
\hline
\end{tabular}

Entre os sujeitos participantes, foi possível verificar desde pesquisadores vinculados a grupos de pesquisa recém-constituídos no ano de 2013, como também grupos formados a mais de 30 anos, que dedicam parte de seus estudos à problemática de resíduos sólidos no Brasil. Fator esse que indica a preocupação de pesquisadores a décadas em relação à geração e gestão dos resíduos sólidos, que perpetua e aumenta 
com o desenvolvimento/crescimento da sociedade moderna responsável pelo aumento de resíduos nos últimos anos.

A formação inicial desses especialistas apresentou a predominância na área das engenharias, principalmente na Engenharia Civil (44,2\%), contudo, pode-se observar a presença de profissionais de várias outras áreas que direcionam seu olhar para questões relacionadas aos resíduos, como direito, arquitetura e urbanismo, administração, oceanografia, química, ciências biológicas, física, geografia, ciências, entre outras (Figura 1).

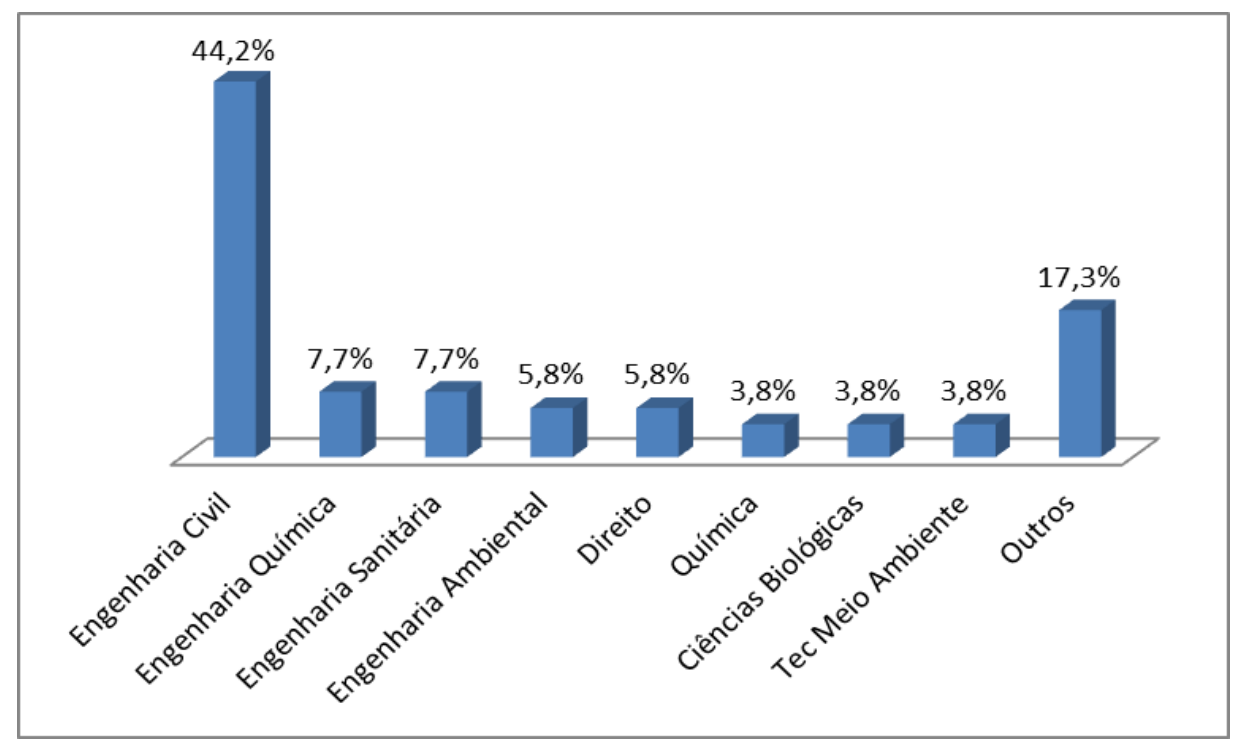

Figura 1 - Distribuição dos especialistas, segundo sua formação na graduação $(n=52)$

Os achados, quanto à graduação desses pesquisadores, revelam uma multidisciplinaridade, que se torna mais visível conforme aumenta a sua formação. Dos 52 participantes, 27 (51,9\%) fizeram especialização. Entre os cursos citados por esses sujeitos, 25,9\% relacionavam-se à área ambiental, 11,1\% à Saúde Pública, 11,1\% à Hidráulica e Saneamento, $11,1 \% \%$ à área da Engenharia Civil, 11,1\% estavam relacionados ao Direito e 29,6\% correspondiam a outras áreas, como, por exemplo, especialização em Gestão de Resíduos Sólidos.

Quanto ao mestrado, somente um sujeito não tinha feito o mestrado por ter realizado o doutorado direto. Assim, todos os especialistas participantes da pesquisa tinham concluído pelo menos o mestrado. A maior área de concentração dos 
mestrados/doutorados realizados por esses sujeitos foi em Hidráulica e Saneamento, Engenharia Civil e Engenharia Ambiental, destacando-se ainda outras áreas como Saúde Pública, Desenvolvimento Urbano, Direito, Geociências, entre outras (Figura 2).

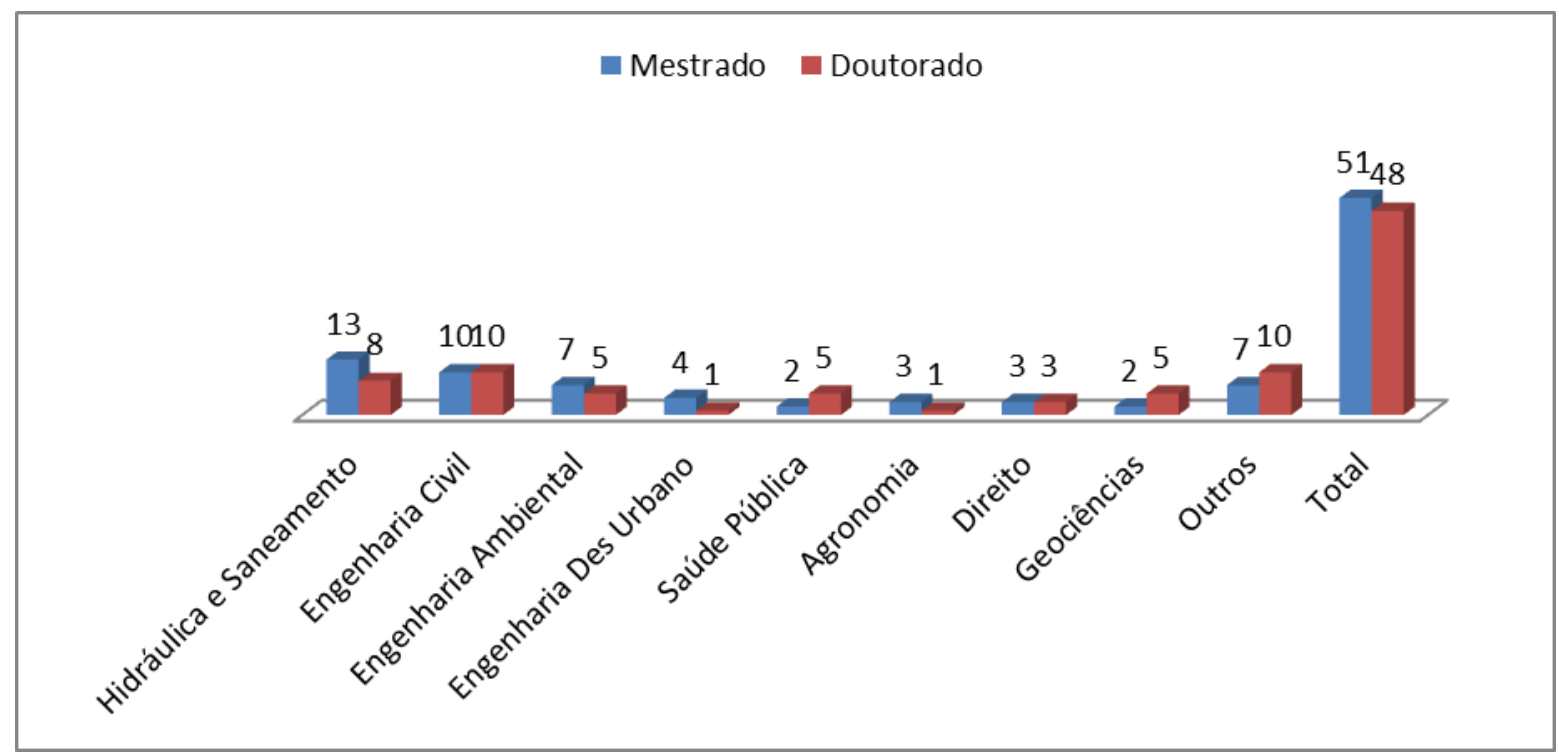

Figura 2 - Distribuição dos especialistas, segundo sua formação no Mestrado $(n=51)$ e Doutorado $(n=48)$

Dos sujeitos participantes, 11 (21,2\%) tinham concluído pós-doutorado, destacando-se áreas de Hidráulica e Saneamento, Engenharia Ambiental, Resíduos Sólidos, entre outras.

Quando indagados sobre a sua atuação em disciplinas relacionadas a resíduos sólidos, 90,4\% dos especialistas indicaram que ministram essas disciplinas em cursos de graduação, especialização, mestrado e/ou doutorado. Dentre essas disciplinas foram citadas gerenciamento/gestão de resíduos, manejo e tratamento de resíduos, gestão e planejamento ambiental, sistemas de saneamento, dentre outras.

\section{CONCLUSÂO}

A publicação da Política Nacional dos Resíduos Sólidos, depois de mais de 20 anos de discussão, trouxe ao Brasil uma nova perspectiva frente às problemáticas relacionadas aos resíduos. A consecução das metas apresentadas por essa política coloca novos desafios para os municípios brasileiros. 
O comprometimento de especialistas na área de resíduos para a realização de novas pesquisas e construção de indicadores de sustentabilidade para a gestão de resíduos corresponde a um fator fundamental que traz subsídios aos gestores para organizar e promover ações em seus municípios e/ou estabelecimentos.

Os dados obtidos, nesta investigação, demonstram que pesquisadores de diferentes regiões brasileiras dedicam seus esforços e estudos no desenvolvimento de pesquisas na área de resíduos. Destaca-se que essas pesquisas são realizadas em diversas frentes, como na gestão de resíduos, na área de políticas públicas, nos impactos à saúde e ao ambiente, na área de educação ambiental, e muitas delas têm o envolvimento da sociedade.

Outro fator observado corresponde à diversidade de formação dos especialistas que realizam pesquisas nessa área. Esse fator possibilita o enriquecimento na construção de indicadores na área, por trazer visões diferenciadas para um mesmo problema, incentivando o "repensar" dos especialistas durante o processo. Os grupos de pesquisa dos sujeitos que participaram desta pesquisa encontram-se distribuídos nas cinco regiões do Brasil, localizados em 17 diferentes estados da federação. Essa característica também traz um perfil importante para o processo de construção de indicadores, visto que, os especialistas vivenciam problemas diferentes em relação à questão de resíduos, segundo a sua realidade local.

Também se considera importante 0 fato de haver grupos de pesquisa que direcionam seus estudos há décadas para analisar as implicações e aumento da geração de resíduos nos últimos anos, e o impacto de gestões inadequadas à saúde e ao meio ambiente.

O desafio atual consiste em disseminar as informações encontradas nessas pesquisas entre especialistas e profissionais que estudam e trabalham na área de resíduos para fundamentar a construção de indicadores de sustentabilidade representativos que possam ser utilizados em realidades distintas a fim de auxiliar a tomada de decisões e promover um monitoramento dos recursos investidos e balizar as ações de educação ambiental e as políticas públicas. 


\section{Agradecimentos}

Esta pesquisa teve o apoio financeiro da Fundação de Amparo à Pesquisa do Estado de São Paulo (Fapesp).

\section{REFERÊNCIAS}

BOSSEL, Hartmut. Indicators for sustainable development: theory, method, applications. Winnipeg-Canadá: International Institute for Sustainable Development, 1999. $110 \mathrm{p}$.

BRASIL. Política Nacional dos Resíduos Sólidos. Lei ㄲo 12.305, de 02 de agosto de 2010. Institui a Política Nacional de Resíduos Sólidos. Diário Oficial da União. Brasília, DF, 03 ago. 2010.

CNUMAD. Agenda 21. Curitiba: IPARDES, 2001. 260 p.

FOSCHINI, Regina Célia. Indicadores de Sustentabilidade Urbana. Fórum Ambiental da Alta Paulista, v. 7, n. 4 p. 744-751, 2011.

FREITAS, Henrique; OLIVEIRA, Mirian; SACCOL, Amarolinda Zanela; MOSCAROLA, Jean. O método de pesquisa survey. Revista de Administração, São Paulo, v. 35, n. 3, p. 105-112, jul-set. 2000 .

GUIMARÃES, Roberto Pereira; FEICHAS, Susana Arcangela Quacchia. Desafios na construção de indicadores de sustentabilidade. Ambiente \& Sociedade, Campinas, v. XII, n. 2, p. 307-323, 2009.

INTERNATIONAL INSTITUTE FOR SUSTAINABLE DEVELOPMENT. Developing Ideas. Bellagio Principles. Disponível em: <http://www.iisd.org/didigest/jan97/principles.htm>. Acesso em: 31 ago. 2013.

MALHEIROS, Tadeu Fabrício; PHILIPPI JR, Arlindo; COUTINHO, Sonia Maria Viggiani. Agenda 21 Nacional e Indicadores de Desenvolvimento Sustentável: contexto brasileiro. Saúde e Sociedade, São Paulo, v. 17, n.1, p. 7-20, 2008.

MEADOWS, Donella. Indicators and information systems for sustainable development. South Africa: The Sustainability Institute, 1998. 77 p. 
OLIVEIRA, Maria Lúcia Carneiro; FARIA, Sueli Corrêa. Indicadores de saúde ambiental na formulação e avaliação de políticas de desenvolvimento sustentável. Revista Brasileira de Ciências Ambientais, São Paulo, v. 11, p. 16-22, dez. 2008.

PAPINI, Solange. Vigilância em Saúde Ambiental: uma nova área de ecologia. 2 ed. São Paulo: Atheneu Editora, 2012. 205 p.

RABELO, Laudemira Silva; LIMA, Patrícia Verônica Sales. Indicadores de sustentabilidade: a possibilidade da mensuração do desenvolvimento sustentável. Revista Eletrônica do Prodema, Fortaleza, v. 1, n.1, p 55-76, dez. 2007.

UNITED NATIONS. Department of Economic and Social Affairs. Indicators of sustainable development: guidelines and methodologies. (Third ed.). New York, United Nations, 2007. 